\title{
Cognitive Enhancement of Sport Technique Learning by Sleep: A Systematic Review
}

\author{
Antonio Cicchella ${ }^{1}$
}

Received: 17 June 2020 / Revised: 30 July 2020 / Accepted: 3 September 2020 / Published online: 14 September 2020

(c) The Author(s) 2020

\begin{abstract}
Purpose The importance of sleep in enhancing the consolidation of memory for movements has been demonstrated. A possible application of sleep manipulation to sport techniques learning is also potentially helpful for training.

Method Eligibility criteria encompass the group of studies (cross-sectionals and interventions) on humans correlating sleep with physical performance. A search on the topic was performed in Medline.

Results After a review of sleep organization and memory systems, an overview of literature specific to learning and sport performance is presented. Building on existing literature, it was found that, in the short period after awakening from a night deprived of sleep, a strong activation of CNS happens possibly due to increased catecholamines secretions. This is thought to benefit performance in sports of short duration where the nervous component is predominant, such as jumping and sprinting. On the contrary, complex cognitive tasks performed in sports such as tennis are instead impaired by sleep deprivation. The same applies for maximal performance in endurance running and weightlifting. Placement of a cognitive challenging training before sleep can therefore benefit the acceleration of the learning process of a novel motor task.

Conclusion Cognitive and emotionally challenging tasks promote an increase of deep sleep, due to the stress put on the brain during learning. In conclusion, practice and/or rehearsal of complex techniques before sleep appears to accelerate the learning process, increasing accuracy and decreasing error rate. However, few studies exist on specific sport techniques, and further research is needed.
\end{abstract}

Keywords Sleep $\cdot$ Sport technique $\cdot$ Fatigue $\cdot$ Motor performance $\cdot$ Motor learning

\section{Introduction}

Sleep is a major factor influencing several biological pathways and behaviours. Effects of sleep on performance has been studied for a long time. Existing scientific literature concerning the relationship between sleep and exercise/sport performance refers mainly to chronobiology [1], physical overload [1,2] and the general effects of sleep deprivation on performance [3]. Relationships between sleep, exercise and immune response were also considered, founding a positive correlation between immune system activity and sleep quality. The topic of sleep as a possible influencer of motor learning emerged relatively recently, even if the interest for the role of sleep and sleep organization in various neuromotor skills learning and performance has increased in the last decades [4-7]. The main reason for this renewed interest is the Aserinski and Kleitman discovery of REM (Rapid Eyes Movement) sleep in 1953 [8] and the subsequent search for the role of sleep phases in cognitive processes during night. The aim of this short review is to investigate the state of the actual knowledge about the sleep effect in learning and in modifying complexes motor tasks in sport, and the possible benefits of using sleep manipulation to maximize the learning of sport techniques. The main questions addressed are: what are the effects of technically challenging training on sleep length and quality? Can sleep manipulation accelerate the learning of technically challenging sport tasks? How does sleep affect different sport skills?

Antonio Cicchella

antonio.cicchella@unibo.it

1 Department of Quality of Life Studies, University of Bologna, Via del Pilastro 8, 40127 Bologna, Italy 


\section{Methods}

This article provides a review of the current available published literature regarding sleep and motor learning of sport tasks in athletes and lists factors possibly responsible for this phenomenon. An online literature search was performed using PubMed from inception of the database to March 2020 with the following keywords used in different combinations: "sleep" "motor learning" "sleep stages" "sleep and training" "sleep and mental processes" "sleep restriction," "sleep extension," "performance," "recovery," "fatigue," "stress". The search strategies were combined, and duplicates were removed by Endnote X7 (Clarivate Analytics, previously Thomson Reuters, Philadelphia, PA, US) and manually. All titles and abstracts were carefully read, and relevant articles were retrieved for review. In addition, the reference lists from both original and review articles retrieved were also reviewed. A total of 29 relevant papers were found. Eligibility criteria limited the search to studies performed on humans (animal studies were included only if strictly relevant and related to the topic), and to studies which are related to motor tasks and sport tasks learning and cognitive processes. 29 studies met the eligibility criteria and were included in the review. Of the retrieved papers, 7 were reviews, 11 were intervention studies (sleep manipulation) and 11 were cross sectional studies (association between sleep and tasks). Inclusion criteria were: (i) research conducted with human participants and (ii) original data from randomized clinical trials (RCTs) on sleep and learning with an acute or long-term exercise intervention, or cross sectional. The exclusion criteria were (i) studies written in languages other than English and French, (ii) animal or in vitro studies, (iii) congress or workshop publications, (iv) studies in which no motor tasks were considered (studies on visual or auditory memory). No limits were used concerning the year of publication. The inclusion or exclusion of articles was determined by applying the above criteria on the title and abstract as a first screening and on full texts as a second screening. Case studies were excluded, although the respective references were consulted and integrated into this revision if responding to the above-mentioned criteria. The results, of founding only 29 papers, can be explained with the difficulties to perform sleep studies on athletes in heavy training regimes.

\section{Results}

Heavy physical loads are known to enhance deep sleep, causing the immediate beginning of REM phases after the subject falls asleep, and are thought to promote the nocturnal release of growth hormone, which is believed to stimulate memory consolidation via its impact on protein synthesis [9]. Effects of training on sleep duration and sleep phases, are summarized in Table 1.

A brief recall of memory structure is necessary to better understand the role of sleep in memory consolidation. The most currently accepted definition of memory systems is modelled after Squire [10] and Tulving [11]. The main subdivision of this model is between declarative and procedural memory. Declarative memory refers to consciously accessible memories for facts and events, while procedural memory can be defined as a system dedicated to the encoding, storage and retrieval of the procedure subtending perceptual-motor, perceptual-verbal and cognitive skills. This system is also commonly defined as implicit memory [10, 11]. REM (also defined "paradoxical" because of the quickness of EEG traces registered on the scalp) sleep is recognized to have an important role in cognitive processes of memory consolidation that happens during night both for semantic and declarative memory and for procedural memory [12-14]. The second part of the night (early hours of the morning) is known to be the period with higher REM phases content. Experimental evidence confirm that REM windows increase in duration during night sleep after the exposition to challenging motor tasks $[7,12]$. The same behaviour was observed in humans [7]. During intensive training for a technically challenging and stressing task (learning trampoline diving by nonexpert divers), subjects showed an increment in duration of REM phases during night after training [7]. In addition to REM, stage 2 (or deep sleep) seems to play a role in cognitive processes that happens during sleep. Stage 2 sleep occurs primarily in the early hours of the morning, and its deprivation has shown to impair the learning of procedural motor tasks [14-16]. However, these results were obtained mostly selective sleep deprivation studies. A limit of selective deprivation studies is that they might induce modification of sleep patterns and cause emotional and attentional disorders and disturbances in biological

Table 1 Effect of training on sleep (REM: rapid eyes movement sleep, SWS: slow waves sleep)

\begin{tabular}{llll}
\hline References & Sleep duration & REM & SWS \\
\hline Torsvall [21] & & $\uparrow$ & $\uparrow$ \\
Buchegger [7] & & $\uparrow$ & \\
Hennevin [12] & & $\uparrow$ & \\
O’Connor [3] & $\uparrow$ (Moderate training) & & \\
Shephard [1] & $\uparrow$ (Moderate training) & & \\
Urhausen [2] & $\downarrow$ (Heavy training) & & $\uparrow$ \\
Huber [20] & & $\uparrow$ & \\
Mah [28] & & $\uparrow$ &
\end{tabular}


rhythms, influencing the results [15]. The time placement of the sleep periods relatively to motor task training is a primary factor influencing task consolidation in memory [16]. Sleep placed immediately after task learning improves memory consolidation, especially if sleep occurs in the second half of the night (higher percent of REM sleep and stage 2 incomparison with the first half). The second half of the night seems thus to be the most important period for the progress of the neurochemical processes involved in memory strengthening and is linked to growth hormone release [9]. In memory consolidation studies, the sleep effect is independent [16] of whether sleep is placed during daytime or night-time. Sleep immediately after practice has proven to enhance speed of sequence performance of a fingers opposition task, by average of $33.5 \%$, and reduced error rate by $30.1 \%$ as compared with corresponding intervals of wakefulness [7]. If the sleep period occurs not immediately after practice, but after several hours after the learning period, the effect of retention was less effective [16]. If the sleep period is placed immediately after the learning task, the retention effect is larger than if the sleep is placed after some hour from the task learning [16]. This effect can also be due to the absence of interfering tasks happening between the time of task learning and the time when the subject falls asleep. Data on a sample of one-night completely sleepdeprived subjects, confirm these results [17]. In this study the accuracy (number of errors in a time-constrains key pressing sequence) was influenced by the effect of sleep deprivation, but was not improved significantly by sleep. After a night of complete sleep deprivation, the error rate increased by $3 \%$. Error reduction after a night of complete sleep is not statistically significant. Velocity (number of exact matches) in our sample shows an increase of $8.1 \%$. after a night of (missing part), while other studies found a $33.5 \%$ increase [18] after partial deprivation. After a night of sleep deprivation, we observed only a slight decrease (1\%) of the velocity. The "sleep effect" thus seems to be of lower magnitude and seems to affect only the velocity of performance of the task but not its accuracy. Given that we used the simplest sequence of finger tapping with respect to previous studies [16], we expected a greater sleep effect on the results. Another explanation for the smaller improvement after total sleep deprivation can be the potent stimulation of the adeno-cortical axis due to stress. A limit for this kind of studies is they are limited to segmental movements as finger tapping tasks. The transferability of this results on training situation is questionable due to the many confounding variables affecting training, where is difficult to separate physical and cognitive fatigue. The question how the learning of more complex movements is influenced by sleep organization remains unresolved, even though in the last years some research addressed this topic.

\subsection{Memory Systems and Learning a Task}

Segmental movements learning for performance in training conditions requires more time to be effective and individual differences also play an important role [18]. Procedural memory is the main system in which motor learning processes are inscribed $[10,11]$ and, as previously reported, increase of REM sleep duration after physical training has been observed in both animals and humans [12]. This aftereffect finding suggest REM sleep plays a fundamental role in the consolidation of motor memory traces. Stimulation during sleep assumes a great interest for conditioning. Tasks performed during awakening periods during nigh time, conflicting or not conflicting with the task learned the evening before sleep, has been shown to damage or to reinforce memory traces [19]. Awareness during learning is however a crucial factor for memory traces formation during night conditioning and it is a factor difficult to assess [19]. There are also robust and persistent findings about the reliance of procedural non declarative memory on sleep [10]. For instance, the sleep effect on memory consolidation on novel acquired motor tasks seems to be specific to the motor sequence learned with the specific hand used to perform the finger tapping tasks [16].

Learning a task before sleep (a mental rotation task) increases slow waves activation (SWA) in selected arrays of neurons in the brain, (Broadman brain area 40 and 7 in the right hemisphere, known to be involved in spatial tasks). The amount of SWA was found to be correlated with the improvement in the task after sleep [20], through synaptic consolidation happening during sleep. SWA increased after sleep onset for the first 90 min by about $25 \%$. These results help relate fine motor task learning with SWA. After an emotionally challenging (but not physically fatiguing in terms of elevated heart rate) gross motor task learning, REM sleep phase appear to increase in length [7]. REM sleep is the dream phase of sleep; thus, this study confirms the triggering effects of emotion during daytime on nocturnal REM length and this is the case of sport competitions. REM sleep increases also after a fatiguing running endurance race and is associated with catecholamine release. Spectral analysis (FFT) of the EEG showed an increase of total power density as well as of power in the delta band after the race. The increase was statistically significant also within SWS [21]. In the learning process, when a subject achieves mastery in a cognitive task (e.g., the task of the Tower of Hanoi) the REM sleep phase also increases in length [28]. This study is in accord with the related study of trampoliners [7]. It is unclear if the modifications of sleeps phases are attributable to increased cognitive or increased emotional task above 
the basal subjective level. One issue is that basal cognitive levels for subjects involved in sleep and learning studies is difficult to assess and is almost never provided in the literature. A hypothesis is that practice placed immediately prior to sleep (even pure rehearsal tasks) triggers mental processes, stimulating neurons to continue to "work" on the last stimuli received prior to sleep [22]. Such practice, for example looking at videos of techniques, could be of benefit for the learning and/or correction of sport techniques. Higher mental processes, such as the influence of sleep on decision making, can be defined as a higher cognitive ability, and is fundamental in sports. This influence has been studied [23] and an effect was found. It has been shown that sleep impairs decision-making involving the unexpected, innovation, revising plans, competing distractions, and effective communication [23], all qualities of maximal importance in sport.

\subsection{Sleep and Sport Techniques}

In sport, very few studies dealt with psychomotor vigilance, fine motor tasks (e.g., skills) and gross motor tasks (e.g., conditioning) $[24,30]$. In a very comprehensive cross-sectional study of Knufinke et al. [24], sleep time was found to be positively associated with reaction time. The longer the sleep, the faster the reaction time to a simple visual stimulus (such as pressing a button at the appearance of a red signal on a computer screen). Reduction of reaction time was strongly associated with more sleep, accounting for $5 \mathrm{~ms}$ decrease for every additional hour of sleep. No association was found between sleep quality (awakening, time to fall asleep and sleep stages) and reaction time. Athletes who sleep less were found to have better gross motor skills performance of maximal sprint, vertical jump, and constant power test in cycling [24], while no association was found with fine motor skills (shooting, dribbling and technical bike skills) [24]. Also sleep quality in this study was not associated with better gross motor skills performance. In longer exercises where the aerobic system is hardly challenged, the performance of the task is impaired by shorter sleep [25]. After partial sleep deprivation (awakening in the middle of the night) performance on the laboratory bike was impaired, with increased heart rate, ventilation and $\mathrm{VE} / \mathrm{VO} 2$ ratio at submaximal intensities ( $75 \%$ of the $\mathrm{VO} 2$ max). Maximal workload and oxygen consumption decreased at maximal work, after the night of partial sleep deprivation as compared to the control. These findings suggest that acute sleep loss may contribute to alter the endurance performance by impairment of aerobic pathways. Two studies were found that analysed the effects of sleep shortening on the specific task of tennis serve, which requires great accuracy. Sleep restriction of 33\% over normal sleep time in test subjects $(5 \mathrm{~h})$ showed a significant impairment in the tennis serve accuracy [26] while extension of sleep improves the same task accuracy [27]. Accuracy is thus improved by longer sleep. Data on sleep quality were provided in this study, showing no association between sleep quality and increased accuracy. Throwing accuracy was also tested in basketball players. Extension of habitual sleep time of $100 \mathrm{~min}$ in collegiate basketball players determined an increase of sprint time, throwing accuracy ( $+9 \%$ in the free throw), and a decrease in reaction time, together with decreased perceived fatigue and increased perceived vigour [28]. Partial sleep loss ( $3 \mathrm{~h}$ per day over a 3 -day period) does not influence maximal biceps curl but a significant decreasing effect was noted on maximal bench press, leg press, and dead lift in gym-trained subjects [29]. Where large muscle masses are involved, the effect of sleep deprivation is more evident, probably due to a summation effect, not visible in exercise involving smaller muscles with less nervous activation.

\section{Conclusions and Recommendation for Practice}

In this brief review, studies dealing with sleep effect on motor and sport techniques shows that sleep organization can be usefully employed to ameliorate the learning of a sport technique. There is strong evidence that prolonging and improving sleep quality improves performance in some tasks, while shortening the sleep improves it for other tasks. The effect of sleep prolongation can be due to the restoration, contrasting the chronic sleep deprivation of contemporary life, as clearly addressed by some authors [30]. Restoration of nervous system functions increases vigilance, awareness and ultimately accuracy of some sport task.

Maximal and larger muscle mass efforts in weightlifting and endurance benefit from longer sleep time while less challenging exercises (e.g., biceps curl) are not influenced by shortened sleep ( $3 \mathrm{~h}$ ). Also, this factor can be attributed to restoration of CNS with sleep and should be considered by coaches.

Short duration tasks (sprinting, jumping) are shown to improve with less sleep, temporarily increasing CNS activation, while technical skills are not influenced. This phenomenon is well known to coaches, which recommend awaking at least 4 or $5 \mathrm{~h}$ before a competition if the race is placed in the morning. Accuracy in tennis serve is improved by longer sleep and impaired by sleep deprivation. Learning of a given technique can benefit from the practice and rehearsal just prior the evening of sleep before the task, thus avoiding memory conflicts and stimulating overnight memory consolidation. Studies in sport support the finding of simplest motor tasks, such as fingers tapping. However, there is a paucity of literature about sleep and sport cognitive aspects, and more studies are necessary in selected sports techniques 
to test the effectiveness of sleep manipulation in specific sport technique learning and improvement. General health of athletes and acceleration in learning benefit from a better sleep. In addition, sleep can be manipulated, activating the CNS with early awakening and shorter sleep (tonic effect of sleep) to stimulate the performance, while during training it emerges from literature to increase sleep to improve recovery and learning (phasic effect of sleep).

Funding Open access funding provided by Alma Mater Studiorum - Università di Bologna within the CRUI-CARE Agreement. The paper was funded by the author fund for basic research of University of Bologna.

\section{Compliance with Ethical Standards}

Conflict of interest The author declare no conflict of interests.

Open Access This article is licensed under a Creative Commons Attribution 4.0 International License, which permits use, sharing, adaptation, distribution and reproduction in any medium or format, as long as you give appropriate credit to the original author(s) and the source, provide a link to the Creative Commons licence, and indicate if changes were made. The images or other third party material in this article are included in the article's Creative Commons licence, unless indicated otherwise in a credit line to the material. If material is not included in the article's Creative Commons licence and your intended use is not permitted by statutory regulation or exceeds the permitted use, you will need to obtain permission directly from the copyright holder. To view a copy of this licence, visit http://creativecommons.org/licenses/by/4.0/.

\section{References}

1. Shephard RJ, Shek PN. Interactions between sleep, other body rhythms, immune responses, and exercise. Can J Appl Physiol. 1997;22:95-116. https://doi.org/10.1139/h97-009.

2. Urhausen A, Kindermann W. Diagnosis of overtraining: what tools do we have? Sports Med. 2002;32:95-102. https://doi. org/10.2165/00007256-200232020-00002.

3. O'Connor PJ, Youngstedt SD. Influence of exercise on human sleep. Exerc Sport Sci Rev. 1995;23:105-34.

4. Karni A, Meyer G, Rey-Hipolito C, et al. The acquisition of skilled motor performance: fast and slow experience-driven changes in primary motor cortex. Proc Natl Acad Sci USA. 1998;95:861-8. https://doi.org/10.1073/pnas.95.3.861.

5. Doyon J, Korman M, Morin A, et al. Contribution of night and day sleep vs simple passage of time to the consolidation of motor sequence and visuomotor adaptation learning. Exp Brain Res. 2009;195:15-26. https://doi.org/10.1007/s00221-009-1748-y.

6. Reilly T, Edwards B. Altered sleep-wake cycles and physical performance in athletes. Physiol Behav. 2007;90:274-84. https://doi. org/10.1016/j.physbeh.2006.09.017.

7. Buchegger J, Meier-Koll A. Motor learning and ultradian sleep cycle: an electroencephalographic study of trampoliners. Percept Mot Skills. 1988;67:635-40. https://doi.org/10.2466/ pms.1988.67.2.635.
8. Aserinsky E, Kleitman N. Regularly occurring periods of eye motility, and concomitant phenomena, during sleep. Science. 1953;118:273-4. https://doi.org/10.1126/science.118.3062.273.

9. Touitou Y, Haus E. Biological Rhythms and Aging. In: Touitou Y, Haus E, editors. Biological rhythms in clinical and laboratory medicine. Berlin: Springer; 1992. p. 188-207.

10. Squire LR, Zola SM. Structure and function of declarative and nondeclarative memory systems. Proc Natl Acad Sci USA. 1996;93:13515-22. https://doi.org/10.1073/pnas.93.24.13515.

11. Tulving E. How many memory systems are there? Am Psychol. 1985;35:335-43. https://doi.org/10.1037//0278-7393.15.1.31.

12. Hennevin E, Hars B, Maho C, Bloch V. Processing of learned information in paradoxical sleep: relevance for memory. Behav Brain Res. 1995;69:125-35. https://doi.org/10.1016/01664328(95)00013-j.

13. Hennevin E, Hars B, Maho C, Bloch V. Augmentation du sommeil paradoxal provoquée par l'acquisition, l'extinction et la reacquisition d'un apprentissage à reinforcement positif. Brain Res. 1974;70:38-54. https://doi.org/10.1016/0006-8993(74)90210-8.

14. O'Connor PJ, Youngstedt SD. Influence of exercise on human sleep. Exerc Sport Sci Rev. 1995;23:105-34.

15. Smith C. Sleep states and memory processes in humans: procedural versus declarative memory systems. Sleep Med Rev. 2001;5:441-56. https://doi.org/10.1053/smrv.2001.0164.

16. Korman M, Doyon J, Doljansky J, Carrier J, Dagan Y, Karni A. Daytime sleep condenses the time course of motor memory consolidation. Nat Neurosci. 2007;10:1206-13. https://doi. org/10.1038/nn1959.

17. Walker MP, Stickgold R. Sleep, memory, and plasticity. Annu Rev Psychol. 2006;57:134-66. https://doi.org/10.1146/annurev.psych .56 .091103 .070307 .

18. Mazzetti M, Plazzi G, Campi C, Cicchella A, Mattarozzi K, Tuozzi G, Vandi S, Vignatelli L, Cipolli C. Sleep-dependent consolidation of motor skills in patients with narcolepsy-cataplexy. Arch Ital Biol. 2012;150:185-93. https://doi.org/10.4449/aib. v150i2/3.1412.

19. Walker MP, Brakefield T, Hobson JA, Stickgold R. Dissociable stages of human memory consolidation and reconsolidation. Nature. 2003;375:616-20. https://doi.org/10.1038/nature01930.

20. Huber R, Ghilardi MF, Massimini M, Tononi G. Local sleep, and learning. Nature. 2004;430:78-81. https://doi.org/10.1038/natur e02663.

21. Torsvall L, Akerstedt T, Lindbeck G. Effects on sleep stages and EEG power density of different degrees of exercise in fit subjects. Electroencephalogr Clin Neurophysiol. 1984;57:347-53. https:// doi.org/10.1016/0013-4694(84)90158-5.

22. Wagner U, Gais S, Haider H, et al. Sleep inspires insight. Nature. 2004;427:352-5. https://doi.org/10.1038/nature02223.

23. Harrison Y, Harrison Y, Horne JA. The impact of sleep deprivation in decision making: a review. J Exp Psychol Appl. 2000;2000(6):236-49. https://doi. org/10.1037//1076-898x.6.3.236.

24. Knufinke M, Nieuwenhuys A, Maase K, et al. Effects of natural between-days variation in sleep on elite athletes' psychomotor vigilance and sport-specific measures of performance. J Sports Sci Med. 2018;17:515-24.

25. Mougin F, Davenne D, Simon-Rigaud ML, Renaud A, Garnier A, Magnin P. Perturbations de la performance sportive après privation partielle de sommeil [Disturbance of sports performance after partial sleep deprivation]. C R Seances Soc Biol Fil. 1989;183:461-6 (in French, English abstract available).

26. Reyner LA, Horne JA. Sleep restriction and serving accuracy in performance tennis players, and effects of caffeine. Physiol Behav. 2013;120:93-6. https://doi.org/10.1016/j.physbeh.2013.07.002. 
27. Schwartz J, Simon RD. Sleep extension improves serving accuracy: a study with college varsity tennis players. Physiol Behav. 2015;151:541-4. https://doi.org/10.1016/j.physbeh.2015.08.035.

28. Mah CD, Mah KE, Kezirian EJ, Dement WC. The effects of sleep extension on the athletic performance of collegiate basketball players. Sleep. 2011;34:943-50. https://doi.org/10.5665/SLEEP .1132.

29. Reilly T, Piercy M. The effect of partial sleep deprivation on weight-lifting performance. Ergonomics. 1994;37:107-15. https ://doi.org/10.1080/00140139408963628.
30. Walker MP. A societal sleep prescription. Neuron. 2019;103(4):559-62. https://doi.org/10.1016/j.neuro n.2019.06.015.

Publisher's Note Springer Nature remains neutral with regard to jurisdictional claims in published maps and institutional affiliations. 